Original Article

\title{
Immediate effects of rhythmic auditory stimulation on gait in stroke patients in relation to the lesion site
}

\author{
Naomi Kobinata, MA ${ }^{1)^{*}}$, Mai Ueno, BA ${ }^{1)}$, Yukihito Imanishi, MD, $\mathrm{PhD}^{2 \text { ), }}$ \\ Hideto YoshiKaWA, MD2) \\ 1) Department of Rehabilitation, Yoshikawa Hospital: 98 Ikejiri, Kishiwada, Osaka 596-0813, Japan \\ 2) Department of Medicine, Yoshikawa Hospital, Japan
}

\begin{abstract}
Purpose] Rhythmic auditory stimulation has been used in gait training for stroke patients. However, few studies have investigated its effects in relation to lesion sites. Therefore, this study examined the immediate effects of rhythmic auditory stimulation on gait in stroke patients with lesions in different regions. [Subjects and Methods] One hundred and five patients were recruited and divided into five groups according to the lesion site: cerebellum, pons and medulla, thalamus, putamen, and corona radiata. During training, participants walked to an auditory, continuous rhythmic beat, which was set to each individual's cadence. [Results] Pre- versus post-test measures revealed significant increases in velocity and stride length in the cerebellum, pons and medulla, and thalamus groups. Although the putamen and corona radiata groups demonstrated increases in velocity and stride length, the increases were not significant. [Conclusion] Rhythmic auditory stimulation was effective in facilitating the prediction of motor timing and gait rhythm in stroke patients with lesions in the cerebellum, pons and medulla, and thalamus, which are associated with impairment of the timing mechanism.

Key words: Rhythmic auditory stimulation, Gait training, Stroke
\end{abstract}

(This article was submitted Mar. 4, 2016, and was accepted May 23, 2016)

\section{INTRODUCTION}

Gait training with auditory cues using metronome clicks or music is known to improve gait in Parkinson's disease $\mathrm{e}^{1-4)}$, in which striatal dopamine decreases due to degeneration and depletion of dopamine neurons in the substantia nigra. Dopamine depletion causes cognitive disturbances, sleep difficulties, and various movement problems including gait disorders such as freezing of gait, festination, and decrease in velocity and stride length ${ }^{5)}$. To improve gait of patients with Parkinson's disease, external information, such as horizontal stripe design on the floor and metronome beats, helps elicit motor responses by bypassing the basal ganglia ${ }^{6}$. Thaut et al. reported that patients with Parkinson's disease who had gait training with rhythmic auditory stimulation (RAS) had significantly improved gait velocity, stride length, step cadence and timing of EMG patterns in the anterior tibialis and vastus lateralis muscles compared to those without RAS ${ }^{1)}$. Hayashi reported that some gait improvement in Parkinson's disease can be achieved by passively listening to music without actual gait training ${ }^{3}$. The disturbed internal rhythm formation could be corrected by external auditory rhythm, which helps achieve a stable gait rhythm.

Recently, RAS has been found to be highly effective in gait training for stroke patients and for those with Parkinson's disease $^{7)}$. A systematic review of interventions for gait coordination following stroke revealed that auditory cueing is one of the most promising approaches in gait training ${ }^{7)}$. According to Thaut et al., in stroke patients, gait training with RAS significantly increased velocity and stride length and reduced electromyographic amplitude variability of the gastrocnemius muscle ${ }^{8)}$. In a study comparing RAS with neurodevelopmental therapy, Bobath based training, for stroke rehabilitation,

\footnotetext{
*Corresponding author. Naomi Kobinata (E-mail: n-kobinata@yoshieikai.com)

(C)2016 The Society of Physical Therapy Science. Published by IPEC Inc.

This is an open-access article distributed under the terms of the Creative Commons Attribution Non-Commercial No Derivatives (by-nc-nd) License $<$ http://creativecommons.org/licenses/by-nc-nd/4.0/>.
} 
Table 1. Participant characteristics

\begin{tabular}{lccccccrr}
\hline \multirow{2}{*}{ Lesion site } & \multirow{2}{*}{ Subject, $\mathrm{n}$} & \multicolumn{2}{c}{ Gender, $\mathrm{n}$} & \multirow{2}{*}{ Age (years) } & \multicolumn{2}{c}{$\begin{array}{c}\text { Time post- } \\
\text { stroke, day }\end{array}$} & \multicolumn{2}{c}{ Stroke type, $\mathrm{n}$} \\
& & Male & Female & & CH & CI \\
\hline Cerebellum & 20 & 15 & 5 & $71.3 \pm 9.5$ & $40.8 \pm 30.6$ & 12 & 8 \\
Pons and medulla & 26 & 21 & 5 & $67.4 \pm 10.9$ & $38.4 \pm 22.8$ & 3 & 23 \\
Thalamus & 22 & 18 & 4 & $64.0 \pm 9.2$ & $61.0 \pm 33.0$ & 18 & 4 \\
Putamen & 18 & 11 & 7 & $64.3 \pm 13.4$ & $42.7 \pm 19.5$ & 15 & 3 \\
Corona radiata & 19 & 12 & 7 & $72.8 \pm 9.4$ & $39.2 \pm 23.2$ & & 19 \\
\hline Total/Mean & 105 & 77 & 28 & $67.9 \pm 11.0$ & $44.37 \pm 27.5$ & 48 & 57 \\
\hline
\end{tabular}

$\mathrm{CH}$ : cerebellar hemorrhage; CI: cerebellar infarction

greater improvement was seen in velocity, stride length, cadence, and symmetry with RAS than with the other approach ${ }^{9)}$. These findings show that RAS is an effective intervention in gait training following stroke.

However, the effect of RAS on specific regions of the brain and its influence on motor output is not fully understood ${ }^{10)}$. Few studies have investigated the effects of RAS in relation to the lesion site in stroke patients ${ }^{11)}$. Therefore, the purpose of this study was to investigate the neurological effects of RAS on gait in stroke patients with lesion in different regions (i.e., the cerebellum, pons and medulla, thalamus, putamen, and corona radiata).

\section{SUBJECTS AND METHODS}

Between April 2006 and June 2011, we included 105 inpatients from the post-acute rehabilitation unit. Participant characteristics are summarized in Table 1 . All subjects were classified stage 3 or above on the Brunnstrom recovery scale of the lower limb (i.e., lower limb spasticity and voluntary control through basic limb synergy $)^{12)}$.

All patients understood the purpose of this study and provided written informed consent prior to participation in the study in accordance with the ethical standards set by the Declaration of Helsinki.

Before RAS, each participant completed a 10-meter walk without RAS (pre-test), after which baseline data for gait cadence, velocity, and stride length were recorded. Then each participant walked to the auditory rhythmic cues at a frequency that matched his/her baseline cadence, as described by Thaut et $\mathrm{al}^{8,9)}$. The auditory cues were provided by a music therapist who either used metronome beats or played steady beats using a musical rhythm instrument such as a drum or an autoharp. When the participant's gait rhythm became stable and matched the auditory cue, the frequency of the auditory rhythm was gradually increased to achieve the ideal cadence without decreasing velocity and stride length and without losing gait balance. After RAS, each participant completed another 10-meter walk without RAS (post-test), after which final gait parameters were recorded. An appropriate walking aid such as a cane or a walker was used during training and testing if necessary. The whole procedure took about 20 minutes.

Paired t-tests were performed to determine if there was a significant difference between the pre- and post-test gait parameters (i.e., velocity and stride length) within the groups. The data were analyzed using SPSS, version 15, for Windows (SPSS, Chicago, IL, USA), and values of $\mathrm{p}<0.05$ were considered statistically significant.

\section{RESULTS}

As presented in Table 1, participant characteristics across groups were relatively similar.

The velocities in the pre- and post-tests for each group are listed in Table 2. Between the pre- and post-tests, the paired t-test revealed significant increases $(\mathrm{p}<0.05)$ in velocity in the cerebellum, pons and medulla, and thalamus groups. The putamen and corona radiata groups demonstrated increases in velocity. However, the increases were not significant.

The stride lengths in the pre- and post-tests for each group are listed in Table 3. Compared to the pre-test, the paired t-test revealed significant increases $(\mathrm{p}<0.05)$ in stride length in the cerebellum, pons and medulla, and thalamus groups in the post-test. The putamen and corona radiata groups demonstrated increases in stride length. However, the increases were not significant.

\section{DISCUSSION}

In this study, RAS was effective in increasing velocity and stride length in the cerebellum, pons and medulla, and thalamus groups. However, RAS did not influence gait parameters in the putamen and corona radiata groups. These findings suggest that the effects of RAS vary by the lesion site. Therefore, we opine that rehabilitation with RAS is effective in improving predictive motor timing in patients with lesions in specific regions.

The lesion sites that RAS affected were the cerebellum, pons and medulla, and thalamus. Lesions in these areas are associ- 
Table 2. The velocities in the pre- and post-tests according to the lesion sites

\begin{tabular}{lccc}
\hline Lesion site & Pre-test, $\mathrm{m} / \mathrm{min}$ & Post-test, $\mathrm{m} / \mathrm{min}$ & \\
\hline Cerebellum & $31.6 \pm 14.6$ & $37.7 \pm 20.4$ & $*$ \\
Pons and medulla & $36.5 \pm 18.6$ & $39.4 \pm 21.3$ & $*$ \\
Thalamus & $17.7 \pm 10.3$ & $21.0 \pm 13.5$ & $*$ \\
Putamen & $21.1 \pm 14.9$ & $22.8 \pm 16.6$ & \\
Corona radiata & $22.9 \pm 11.9$ & $25.2 \pm 15.7$ & \\
\hline$* \mathrm{p}<0.05$ & & &
\end{tabular}

Table 3. The stride lengths in the pre- and post-tests according to the lesion sites

\begin{tabular}{lccc}
\hline Lesion site & Pre-test, $\mathrm{m}$ & Post-test, $\mathrm{m}$ & \\
\hline Cerebellum & $0.64 \pm 0.23$ & $0.73 \pm 0.33$ & $*$ \\
Pons and medulla & $0.71 \pm 0.24$ & $0.75 \pm 0.26$ & $*$ \\
Thalamus & $0.55 \pm 0.15$ & $0.61 \pm 0.19$ & $*$ \\
Putamen & $0.54 \pm 0.22$ & $0.57 \pm 0.24$ & \\
Corona radiata & $0.57 \pm 0.19$ & $0.6 \pm 0.20$ & \\
\hline *p $<0.05$ & & &
\end{tabular}

ated with motor timing impairment; thus, RAS may compensate for the timing impairment. The cerebellum is known to play a critical role in anticipating and predicting timing ${ }^{13)}$. Lesions in the cerebellum impair motor coordination and affect speed, rhythm, accuracy, and smoothness of motion, such as gait ${ }^{14}$. Along with the cerebellum, the pons and medulla play a role in the timing mechanism ${ }^{15)}$. The pons receives signals from the cerebrum and relays information to the cerebellum. The pontine lesion can disrupt the corticopontocerebellar circuit and cause cerebral ataxia ${ }^{16}$. The medulla is also closely connected to the cerebellum. The inferior olivary nucleus, situated in the medulla, encodes the timing of sensory input and decodes it to the cerebellum by synchronizing the spikes of Purkinje cells with its rhythmic oscillatory activity. Therefore, the inferior olivary nucleus in the medulla has a role in the control of motor timing as a part of the olivo-cerebellar system ${ }^{17)}$. Finally, the thalamus, which directly receives the output from the cerebellum, is another area where a lesion can cause motor timing impairment, cerebellar ataxia, and ataxia induced by the disturbance of the sense of depth ${ }^{18}$. Since RAS affected the velocity and stride length in these groups, RAS seems to compensate for the motor timing impairment, leading to an improvement in gait parameters.

Conversely, RAS did not influence the velocity and stride length in the putamen and corona radiata groups. Lesions in these sites may cause motor timing impairment; however, this is rare. In case of the putamen, impairment in motor timing occurs with bilateral lesions, not unilateral lesions ${ }^{19,20)}$. In case of unilateral putamen lesion, the intact hemisphere may compensate for motor timing in contrast to the unilateral lesions in the cerebellum, pons and medulla, and thalamus. Lesions in the corona radiata may cause an ataxic hemiparesis syndrome with accompanying sensory impairment by disrupting the cerebellocortical pathway ${ }^{21,22}$. However, the occurrence is infrequent and the influence on gait is minimal ${ }^{22,23)}$. As lesions in these regions do not significantly impair motor timing, expectedly, RAS does not significantly influence the gait parameters of patients with lesions in these regions.

Since patient characteristics across groups were relatively similar, it was unlikely that these would have influenced the effects of RAS. However, this study is limited to comparing the pre- and post-test gait parameters within the groups. Further studies, including a control group and performing between group comparisons are needed to clarify group differences.

In conclusion, RAS was effective in facilitating the prediction of motor timing and gait rhythm in stroke patients with lesion in the cerebellum, pons and medulla, and thalamus, which are associated with impairment of the timing mechanism.

\section{REFERENCES}

1) Thaut MH, McIntosh GC, Rice RR, et al.: Rhythmic auditory stimulation in gait training for Parkinson's disease patients. Mov Disord, 1996, 11: 193-200. [Medline] [CrossRef]

2) McIntosh GC, Brown SH, Rice RR, et al.: Rhythmic auditory-motor facilitation of gait patterns in patients with Parkinson's disease. J Neurol Neurosurg Psychiatry, 1997, 62: 22-26. [Medline] [CrossRef]

3) Hayashi A: Music therapy for Parkinson's disease. Neurol Therap, 2007, 24: 703-710 (in Japanese).

4) Lim I, van Wegen E, de Goede C, et al.: Effects of external rhythmical cueing on gait in patients with Parkinson's disease: a systematic review. Clin Rehabil, 2005, 19: 695-713. [Medline] [CrossRef]

5) Takakusaki K: [Function of cortical basal nuclei: pathophysiology of Parkinson's disease]. Nihon Seirigaku Zasshi, 2003, 65: 113-129 (in Japanese). [Medline]

6) Taniwaki T, Okayama A, Yoshiura T, et al.: Functional network of the basal ganglia and cerebellar motor loops in vivo: different activation patterns between self-initiated and externally triggered movements. Neuroimage, 2006, 31: 745-753. [Medline] [CrossRef]

7) Hollands KL, Pelton TA, Tyson SF, et al.: Interventions for coordination of walking following stroke: systematic review. Gait Posture, 2012 , 35: 349-359. [Medline] [CrossRef]

8) Thaut MH, McIntosh GC, Rice RR: Rhythmic facilitation of gait training in hemiparetic stroke rehabilitation. J Neurol Sci, 1997, 151: 207-212. [Medline] [CrossRef]

9) Thaut MH, Leins AK, Rice RR, et al.: Rhythmic auditory stimulation improves gait more than NDT/Bobath training in near-ambulatory patients early poststroke: a single-blind, randomized trial. Neurorehabil Neural Repair, 2007, 21: 455-459. [Medline] [CrossRef]

10) Arita H: Ningensei no nyuro saiensu: zentouzenya, taijyoukai, touhishitsu no seirigaku. Tokyo: Chugai-Igaku, 2011. (in Japanese).

11) Abiru M, Nakano C, Mihara Y, et al.: The effects of neurologic music therapy on gait disturbance in stroke patients of different lesion area. Jpn J Music Ther, 
2010, 10: 20-27 (in Japanese).

12) Brunnstrom S: Movement therapy in hemiplegia: a neurophysiological approach. Philadelphia: Harper \& Row, 1970

13) Ivry RB, Keele SW: Timing functions of the cerebellum. J Cogn Neurosci, 1989, 1: 136-152. [Medline] [CrossRef]

14) Hirano S, Hattori T: Ataxia and decreased muscle tonus. Clin Neurosci, 2005, 23: 1370-1373 (in Japanese).

15) Kawamura K: Nou to seishin: seimei no hibiki. Tokyo: Keio Gijuku Daigaku Shuppankai, 2006 (in Japanese).

16) Schmahmann JD, Ko R, MacMore J: The human basis pontis: motor syndromes and topographic organization. Brain, 2004, 127: 1269-1291. [Medline] [CrossRef]

17) Llinás RR: Inferior olive oscillation as the temporal basis for motricity and oscillatory reset as the basis for motor error correction. Neuroscience, 2009, 162 : 797-804. [Medline] [CrossRef]

18) Hirayama K: Neurological syndrome. Tokyo: Bunkodo, 1971 (in Japanese).

19) Aparicio P, Diedrichsen J, Ivry RB: Effects of focal basal ganglia lesions on timing and force control. Brain Cogn, 2005, 58: 62-74. [Medline] [CrossRef]

20) Coslett HB, Wiener M, Chatterjee A: Dissociable neural systems for timing: evidence from subjects with basal ganglia lesions. PLoS ONE, 2010 , 5: e10324. [Medline] [CrossRef]

21) Wild B, Dichgans J: Cerebellar ataxia in ataxic hemiparesis? A kinematic and EMG analysis. Mov Disord, 1993, 8: 363-366. [Medline] [CrossRef]

22) Caplan LR: Ataxia in patients with brain infarcts and hemorrhages. Handb Clin Neurol, 2012, 103: 147-160. [Medline] [CrossRef]

23) Maeshima S, Dohi N, Kajiwara T, et al.: A clinical study of ataxia hemiparesis due to cerebrovascular disorder. Jpn J Rehabil Med, 1991, 28: 121-126 (in Japanese). [CrossRef] 